\title{
Epidemiology of Antibiotic Resistance in Culture-positive Hospitalized Patients in Selected Hospitals in Khartoum, Sudan
}

Corresponding Author: Nagwa M. El Amin; email:

nagwaelamin@qumed.edu.sa

Received 15 January 2019 Accepted 26 March 2019 Published 31 March 2019

Production and Hosting by Knowledge E

(c) Nagwa M. El Amin et al. This article is distributed under the terms of the Creative Commons

Attribution License, which permits unrestricted use and redistribution provided that the original author and source are credited.

Editor-in-Chief:

Prof. Mohammad A. M. Ibnouf

\author{
Nagwa M. El Amin ${ }^{1}$, Einas A. Osman², Leena Al-Hassan ${ }^{3}$, and \\ Ihab B Abdalrahman ${ }^{4}$ \\ ${ }^{1}$ Qassim College of Medicine, Qassim University, KSA \\ ${ }^{2}$ Africa University, Sudan \\ ${ }^{3}$ Brighton and Sussex Centre for Global Health Research, Brighton, UK \\ ${ }^{4}$ Department of Internal Medicine, University of Khartoum, Sudan
}

\section{Abstract}

Objective: To study the prevelence of antibiotic resistance and the prevalent bacterial isolates in hospitalized patients in Khartoum hospitals.

Materials \& Methods: A cross-sectional prevalence study was carried out during the period of April-November 2015 in Khartoum; 226 bacterial cultures were included. Identification of isolates using standard biochemical tests and antibiotic susceptibilities were determined using disc diffusion method. Results were interpreted according to the standards of the British society of antimicrobial chemotherapy.

Results: Eight bacterial species were isolated: Staphylococcus aureus, Enterococcus faecalis, Streptococcus spp., Klebsiella pneumoniae, Pseudomonas spp., Escherichia coli, Proteus spp., and Acinetobacter spp. S. aureus was the most prevalent, the majority of which were resistant to methicillin/oxacillin (MRSA). Cultures in our study were mainly from urine (36.7\%), blood samples (37.2\%), and wound cultures (19\%) More than $90 \%$ of the tested isolates were resistant to cefuroxime; $54 \%$ and $73.8 \%$ of Gram-positive and Gram-negative isolates, respectively, were resistant to ceftazidime. Furthermore, there was a high meropenem resistance among Gram-negative isolates tested. Multi-resistant Acinetobacter spp. as well as vancomycin-resistant $S$. aureus was isolated. Gram-negative isolates showed good susceptibilities to aminoglycosides as well as ciprofloxacin. However, the high resistance rate to these antibiotics was observed in Gram-positive isolates in these hospitals.

Conclusion: Methicillin-resistant S. aureus was the most prevalent organism. Gramnegative isolates showed good susceptibilities to aminoglycosides and ciprofloxacin. There were high resistance rates to cefuroxime, ceftazidime, and meropenem. Five vancomycin-resistant S. aureus were identified.

Keywords: antibiotic resistance, Khartoum hospitals

\section{G OPEN ACCESS}




\section{Introduction}

The worldwide spread of bacterial resistance to antimicrobial agents may limit the future progress of medicine. A huge environmental antibiotic pressure, resulting from industrial production and marketing of these drugs, has simultaneously contributed to the increase in the diversity of resistant phenotypes, to the selection of the fittest among them, and to the spreading of resistance genes. The antimicrobial resistance is recognized as global crises, and in 2012, WHO Patient Safety launched a book, The evolving threat of antimicrobial resistance - Options for action.

In Sudan, few studies were performed to assess the problem of antibiotic resistance which we expect to be a major one as antibiotics use is not under control, and infection control measures are not regularly practiced in hospitals [1]. The aim of this study was to study the frequency of antibiotic-resistant bacteria and to identify the most prevalent organisms causing hospital infections in four hospitals in Khartoum state. We also studied the prevalence of multi-resistant bacteria in the hospitals included in the study.

\section{Materials and Methods}

Ethical approval was obtained from the Ministry of Health Khartoum, Sudan. A crosssectional study was conducted.

\subsection{Sampling procedure}

The samples included bacteria grown on media. The sample frame composed of all positive cultures during the study period. Systematic random sampling was used to select the sample.

\subsection{Patients and samples}

The study was carried out in four tertiary hospitals in Khartoum, Sudan.

Samples (blood in case of sepsis or from other sites of infection) were collected by the hospital staff upon the development of infection. When bacterial growth was confirmed by the microbiology laboratories at the hospital, the species were identified and stored at $-80^{\circ} \mathrm{C}$ until further use. The isolates were identified using basic biochemical identification tests according to the Clinical and Laboratory Standards Institute [2, 3]. Antibiotic 
susceptibility testing was performed using the disc diffusion method according to the British Society for Antimicrobial Chemotherapy (BSAC) [4, 5].

A database was set up to record the data associated with the bacterial sample.

\section{Results}

During the period between April and November 2015, 622 positive cultures were grown in the four different hospitals under study (Soba University hospital, Omdurman pediatric hospital, Reibat hospital, and Bahri teaching hospital).

Two hundred and twenty-eight isolates were selected by a systematic random stratified method from hospitals' microbiology laboratories.

Isolates were recovered from different clinical samples, collected from hospitalized patients with clinical symptoms and signs of infection.

The phenotypic identification revealed mainly eight bacterial species (spp.) including Staphylococcus aureus, Enterococcus faecalis, Streptococcus spp. (Figure 1), Klebsiella pneumoniae, Pseudomonas spp., Escherichia coli, Proteus spp., and Acinetobacter spp (Figure 2).

Soba university hospital and Reibat hospital laboratories are the only hospitals in our study that performed blood cultures. All four microbiology laboratories processed different clinical samples such as urine, wound swabs, sputum, body fluids, and swabs from the upper respiratory tract. Gram-positive bacteria mainly S. aureus and S. faecalis were the most frequent isolates from all sites of infection. Gram-negative bacilli were mainly isolated from blood and urine cultures.

Analysis of the antibiotic susceptibility pattern among the isolates in the study had shown a high resistance rate to many $\beta$-lactam antibiotics. The highest resistance rate was found to be to amoxicillin, followed by cefoxitin, cefuroxime, and ampicillin, respectively (Tables 1 and 2 ).

Staphylococcus aureus was the most prevalent organism causing infections in all the hospitals studied (Figure 1). The majority of $S$. aureus isolates were resistant to methicillin/oxacillin (MRSA), which indicates their multi-resistant phenotype. Infection with MRSA was highest among Omdurman hospital patients where $80 \%$ of the $S$. aureus isolates were MRSA. Alarmingly, vancomycin-resistant S. aureus (VRSA) isolates were found in two of the hospitals under study and multi-resistant Acinetobacter spp. as well as carbapenem (meropenem)-resistant Gram-negative bacteria were isolated 
in one hospital. Twenty percent of the Gram-negative isolates tested for meropenem susceptibility was resistant to this antibiotic.

Gram-negative isolates from all four hospitals showed good susceptibilities to aminoglycosides such gentamycin and amikacin as well as the fluoroquinolone, ciprofloxacin (Table 2). However, a high resistance rate was observed in Gram-positive isolates in these hospitals (Table 1).

TABLE 1: Antibiotic susceptibility pattern among Gram-positive isolates.

Antibiotic
Vancomycin
Gentamycin
Ceftriaxone
Amikacin
Cefotaxime
Ciprofloxacin
Ceftazidime
Erythromycin
Oxacillin/methicillin
Trimethoprim-sulfamethoxazole
Amoxicillin-clavulanate
Ampicillin

\begin{tabular}{|c|}
\hline \% Susceptible Isolates \\
\hline 92.8 \\
\hline 79.3 \\
\hline 73.9 \\
\hline 67.7 \\
\hline 54.2 \\
59.3 \\
45.9 \\
38.2 \\
38.1 \\
36.7 \\
35.7 \\
25
\end{tabular}

\begin{tabular}{|c|}
\hline \% Resistant Isolates \\
\hline 7.2 \\
\hline 20.7 \\
\hline 26.1 \\
32.3 \\
45.8 \\
40.7 \\
54.1 \\
61.8 \\
61.9 \\
63.3 \\
64.3 \\
75 \\
\hline
\end{tabular}

TABLE 2: Antibiotic susceptibility pattern among Gram-negative isolates.

Antibiotic
Colistin
Amikacin
Meropenem
Gentamycin
Ceftriaxone
Ciprofloxacin
Trimethoprim-
sulfamethoxazole
Ceftazidime
Piperacillin-tazobactam
Cefuroxime
Cefoxitin
Ampicillin/amoxicillin

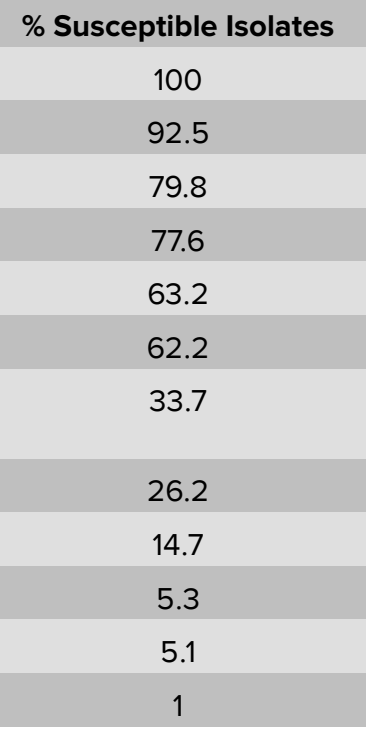

\% Resistant Isolates
0
7.5
20.2
22.4
36.8
37.8
66.3
73.8
85.3
94.7
99




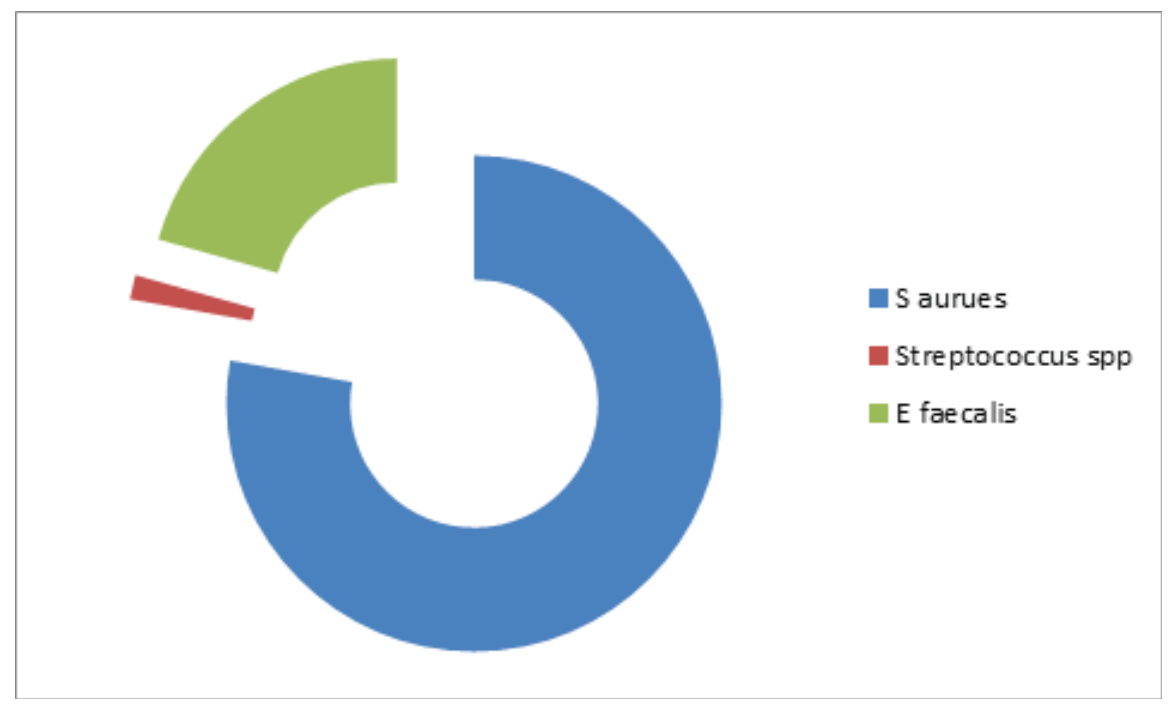

Figure 1: Gram-positive bacteria cultured from hospitalized infected patients over an eight-months period with $S$. aureus being the most common in all four hospitals.

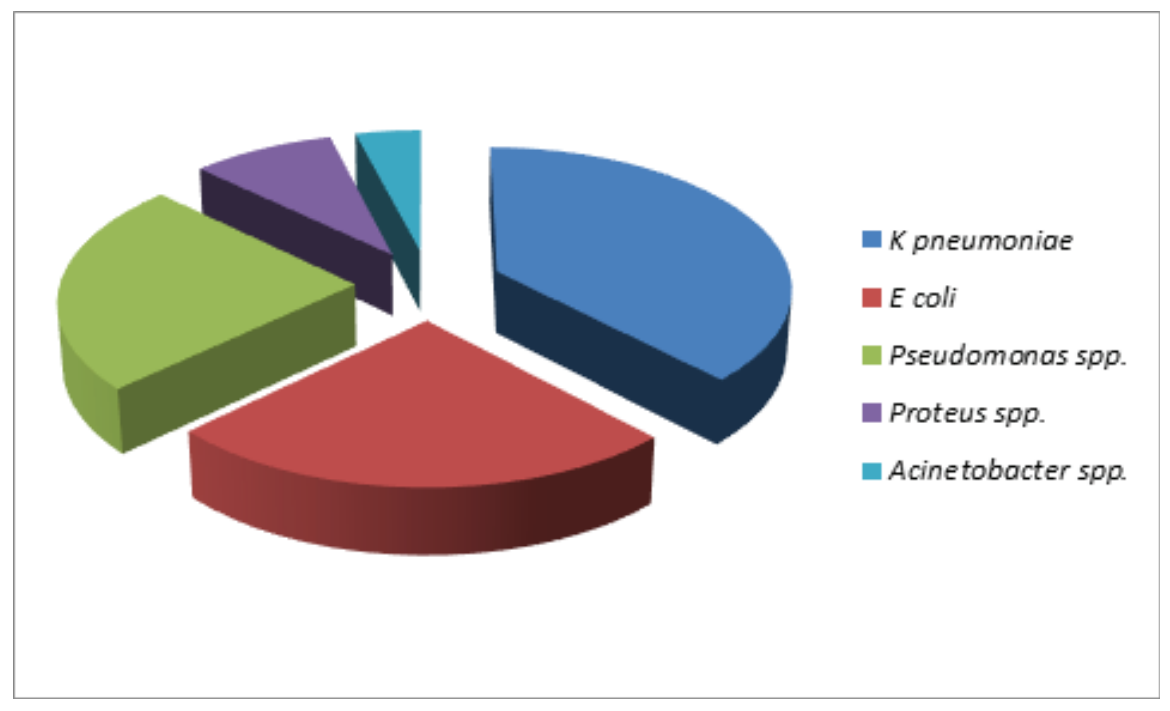

Figure 2: Klebsiella pneumoniae was the most prevalent Gram-negative isolate during the study period and Acinetobacter spp. was the least prevalent.

\section{Discussion}

The majority of positive cultures in this study were isolated from patients with urinary tract infections (UTI) (36.7\%) and blood samples from patients with bloodstream infections. UTI, particularly catheter-associated urinary tract infections (CAUTIs) were shown to account for approximately $40 \%$ of all healthcare-associated infections [7]. Despite studies showing the benefit of interventions for the prevention of CAUTI, the adoption of these practices has not occurred in many hospitals in Sudan.

The most frequent pathogens causing healthcare-associated UTI include $E$. coli, $P$. aeruginosa, enterococci, and Candida albicans; in our study, UTI was mainly caused 
by K. pneumoniae, S. aureus, E. coli, E. faecalis, Pseudomonas spp., and Proteus spp. Studies have shown that both the species distribution and rates of resistance vary considerably among the institutions and regions $[7,8]$.

The second highest culture-positive rate in this study was observed in blood cultures that have very serious implications as shown in former studies. It is well recognized that hospital-acquired severe sepsis was associated with both higher mortality and resource utilization than community-acquired severe sepsis [9].

Cultures from wounds, including surgical site infections (SSIs), were the third frequent positive cultures in this study (19\%). SSIs are a major contributor to the morbidity and mortality in postsurgical care. The risk for SSIs is multifactorial and includes a host of microbial, patient-related, and procedure-related factors [10].

The incidence of SSI was shown to be as high as $20 \%$, depending on the surgical procedure, the surveillance criteria used, and the quality of data collection. In many SSIs, the responsible pathogens originate from the patient's endogenous flora. The causative pathogens depend on the type of surgery; the most commonly isolated organisms are S. aureus, coagulase-negative staphylococci, Enterococcus spp., and E. coli [11]. In the current study, wound infections, including SSIs, were frequently caused by S. aureus, the majority of which were MRSA.

High prevalence of MRSA is a major problem identified in this study and needs to be addressed urgently. Methods such as screening for colonization with MRSA is needed as it has been shown to increase the risk of infection [12-14].

VRSA was identified in two hospitals. The emergence of VRSA emphasizes the high need for programs to prevent the spread of antimicrobial-resistant microorganisms and control the use of antimicrobial drugs in healthcare settings.

Multi-resistant Acinetobacter spp. was isolated in the intensive care unit-hospitalized patients in one of the hospitals studied. Acinetobacter spp. was grown from different infection sites. Since the 1970s, the spread of multidrug-resistant (MDR) Acinetobacter strains among critically ill, hospitalized patients, and subsequent epidemics, have become an increasing cause of concern. Before the 1970s, Acinetobacter infections were mostly post-surgical UTI in patients hospitalized in surgical units. The significant improvement in resuscitation techniques during the last 30 years has changed the types of infection caused by Acinetobacter spp. and since the 1980s, it has spread rapidly among patients in intensive care units $[15,16]$.

The isolates in the present study exhibited high resistance rates to many antibiotics including cephalosporin and carbapenem antibiotics. Resistance to ceftazidime ranged 
from $54.1 \%$ to as high as $73.8 \%$ in some hospitals. The presence of the extended $\beta$ lactamase CTX-M is likely in these isolates $[17,18]$.

The very high rate of resistance to amoxicillin (99\%) amongst the isolates in the current study is very alarming. The probability of spread of a resistant clone should be investigated.

High resistance to meropenem was found in Gram-negative isolates under study that must be handled seriously as carbapenem resistance spreads rapidly and causes challenges in treatment, resulting in high mortality and morbidity rates. It is necessary to observe the use of these antibiotics and to take essential infection control precautions to avoid the spread of this resistance [19-22].

\section{Conclusions}

- Staphylococcus aureus was the most prevalent organism all the hospitals studied. The majority of $S$. aureus isolates were MRSA.

- Gram-negative isolates from all four hospitals showed good susceptibilities to gentamycin and amikacin as well as ciprofloxacin. However, a high resistance rate was observed in Gram-positive isolates in these hospitals.

- There are high resistance rates to cefuroxime and ceftazidime. High carbapenem resistance was found amongst Gram-negative isolates.

- Five VRSA were identified.

\section{References}

[1] Ibrahim, M. E., Bilal, N. E., and Hamid, M. E. (2012). Increased multi-drug resistant Escherichia coli from hospitals in Khartoum state, Sudan. African Health Science, vol. 12, no. 3, pp. 368-375.

[2] Luce, E. (2010). Plastic and reconstructive surgery, in Koneman's Color Atlas and Textbook of Diagnostic Microbiology (sixth edition), vol. 125, pp. 414-415.

[3] Hall, G. S. (2013). Bailey \& Scott's diagnostic microbiology (thirteenth edition). Laboratory Medicine, vol. 44, no. 4, p. e138-e139.

[4] Howe, R. A. and Andrews, J. M. (2012). BSAC standardized disc susceptibility testing method (version 11). Journal of Antimicrobial Chemotherapy, vol. 67, pp. 2783-2784. 
[5] Hudzicki, J. Kirby-Bauer disk diffusion susceptibility test protocol [Internet], pp. 114. Retrieved from: http://scholar.google.com/scholar?hl=en\&btnG=Search\&q=intitle: Kirby-Bauer+Disk+Diffusion+Susceptibility+Test+Protocol\#0.

[6] Shuman, E. K. and Chenoweth, C. E. (2010). Recognition and prevention of healthcare-associated urinary tract infections in the intensive care unit. Critical Care Medicine, vol. 38, no. 8, pp. S373-S379. Retrieved from: http://www.ncbi.nlm.nih. gov/pubmed/20647795.

[7] Bagshaw, S. M. and Laupland, K. B. (2006). Epidemiology of intensive care unitacquired urinary tract infections. Current Opinion in Infectious Diseases, vol. 19, no. 1, pp. 67-71.

[8] Wagenlehner, F. M. E., Cek, M., Naber, K. G., et al. (2012). Epidemiology, treatment and prevention of healthcare-associated urinary tract infections. World Journal of Urology, vol. 30, pp. 59-67.

[9] Page, D. B., Donnelly, J. P., and Wang, H. E. (2015). Community-, healthcare-, and hospital-acquired severe sepsis hospitalizations in the university health system consortium. Critical Care Medicine, vol. 43, no. 9, pp. 1945-1951. Retrieved from: http://content.wkhealth.com/linkback/openurl?sid=WKPTLP:landingpage\&an= 00003246-900000000-97230\%5Cnhttp://www.ncbi.nlm.nih.gov/pubmed/ 26110490\%5Cnhttp://www.pubmedcentral.nih.gov/articlerender.fcgi?artid= PMC4537676

[10] Young, P. Y. and Khadaroo, R. G. (2014). Surgical site infections. Surgical Clinics of North America, vol. 94, pp. 1245-1264.

[11] Owens, C. D. and Stoessel, K. (2008). Surgical site infections: epidemiology, microbiology and prevention. Journal of Hospital Infection, vol. 70, no. 2, pp. 310.

[12] Davis, K. A., Stewart, J. J., Crouch, H. K., et al. (2004). Methicillin-resistant Staphylococcus aureus (MRSA) nares colonization at hospital admission and its effect on subsequent MRSA infection. Clinical Infectious Diseases, vol. 39, no. 6, pp. 776-782.

[13] Gould, I. M. (2007). MRSA bacteraemia. International Journal of Antimicrobial Agents, vol. 30, no. 1, pp. 66-70.

[14] Richard, E. P. (2010). The silent epidemic: CA-MRSA and HA-MRSA. Journal of the American Academy of Orthopaedic Surgeons, pp. 2-4.

[15] Ahoyo, T. A., Bankolé, H. S., Adéoti, F. M., et al. (2014). Prevalence of nosocomial infections and anti-infective therapy in Benin: results of the first nationwide survey in 2012. Antimicrobial Resistance \& Infection Control, vol. 3, no. 17, pp. 2-7. Retrieved 
from: http://download.springer.com/static/pdf/228/art\%3A10.1186\%2F2047-2994-317.pdf?originUrl=http://aricjournal.biomedcentral.com/article/10.1186/2047-2994-317\&token2=exp=1473179291 acl=/static/pdf/228/art\%253A10.1186\%25.

[16] A. M. and E. M. (2013). Antibiotic resistance. Medicine, vol. 41, no. 11, pp. 642-648. Retrieved from: http://ovidsp.ovid.com/ovidweb.cgi?T=JS\&PAGE= reference $\& D=$ emed11\&NEWS $=N \& A N=2013695730$.

[17] Stürenburg, E., Kühn, A., Mack, D., et al. (2004). A novel extended-spectrum $\beta$-lactamase CTX-M-23 with a P167T substitution in the active-site omega loop associated with ceftazidime resistance. Journal of Antimicrobial Chemotherapy, vol. 54, no. 2, pp. 406-409.

[18] Novais, Â., Cantón, R., Coque, T. M., et al. (2008). Mutational events in cefotaximase extended-spectrum? - lactamases of the CTX-M-1 cluster involved in ceftazidime resistance. Antimicrobial Agents and Chemotherapy, vol. 52, no. 7, pp. 2377-2382.

[19] Temkin, E., Adler, A., Lerner, A., et al. (2014). Carbapenem-resistant Enterobacteriaceae: Biology, epidemiology, and management. Annals of the New York Academy of Sciences, vol. 1323, no. 1, pp. 22-42.

[20] Falagas, M. E., Tansarli, G. S., Karageorgopoulos, D. E., et al. (2014). Deaths attributable to carbapenem-resistant Enterobacteriaceae infections. Emerging Infectious Diseases, vol. 20, no. 7, pp. 1170-1175.

[21] Lee, C. R., Cho, I. H., Jeong, B. C., et al. Strategies to minimize antibiotic resistance. International Journal of Environmental Research and Public Health, vol. 10, pp. 4274-4305.

[22] World Health Organization. (2014). The evolving threat of antimicrobial resistance: Options for action. Indian Journal of Medical Research, vol. 139, no. 1, pp. 182-183. Retrieved from: http://www.ijmr.org.in/article.asp?issn=0971-5916;year= 2014; volume=139;issue=1;spage=182; epage=183; aulast=Kapi. 